William Crawley-Boevey

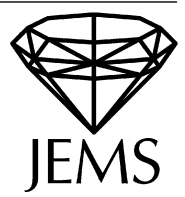

\title{
Kac's Theorem for weighted projective lines
}

Received September 9, 2007 and in revised form October 13, 2008

\begin{abstract}
We prove an analogue of Kac's Theorem, describing the dimension types of indecomposable coherent sheaves (or parabolic bundles) over weighted projective lines in terms of root systems for loop algebras of Kac-Moody Lie algebras. We use a theorem of Peng and Xiao to associate a Lie algebra to the category of coherent sheaves for a weighted projective line over a finite field, and find elements of this Lie algebra which satisfy the relations defining the loop algebra. We use these elements in the proof of our analogue of Kac's Theorem.
\end{abstract}

Keywords. Weighted projective line, parabolic bundle, Kac-Moody Lie algebra, loop algebra, Hall algebra

\section{Weighted projective lines}

Let $K$ be an algebraically closed field, let $\mathbb{P}^{1}$ be the projective line over $K$, let $D=$ $\left(a_{1}, \ldots, a_{k}\right)$ be a collection of distinct points of $\mathbb{P}^{1}$, and let $\mathbf{w}=\left(w_{1}, \ldots, w_{k}\right)$ be a weight sequence, that is, a sequence of positive integers. The triple $\mathbb{X}=\left(\mathbb{P}^{1}, D, \mathbf{w}\right)$ is called a weighted projective line. Geigle and Lenzing [4] have associated to each weighted projective line a category coh $\mathbb{X}$ of coherent sheaves on $\mathbb{X}$, which is the quotient category of the category of finitely generated $\mathbf{L}(\mathbf{w})_{+}$-graded $S(\mathbf{w}, D)$-modules, modulo the Serre subcategory of finite length modules. Here $\mathbf{L}(\mathbf{w})$ is the rank 1 additive group

$$
\mathbf{L}(\mathbf{w})=\left\langle\vec{x}_{1}, \ldots, \vec{x}_{k}, \vec{c} \mid w_{1} \vec{x}_{1}=\cdots=w_{k} \vec{x}_{k}=\vec{c}\right\rangle
$$

partially ordered, with positive cone $\mathbf{L}(\mathbf{w})_{+}=\mathbb{N} \vec{c}+\sum_{i=1}^{k} \mathbb{N} \vec{x}_{i}$, and

$$
S(\mathbf{w}, D)=K\left[u, v, x_{1}, \ldots, x_{k}\right] /\left(x_{i}^{w_{i}}-\lambda_{i} u-\mu_{i} v\right),
$$

with grading $\operatorname{deg} u=\operatorname{deg} v=\vec{c}$ and $\operatorname{deg} x_{i}=\vec{x}_{i}$, where $a_{i}=\left[\lambda_{i}: \mu_{i}\right] \in \mathbb{P}^{1}$. Geigle and Lenzing showed that $\operatorname{coh} \mathbb{X}$ is a hereditary abelian category with finite-dimensional Hom and Ext spaces. The free module gives a structure sheaf $\mathcal{O}$, and shifting the grading gives twists $E(\vec{x})$ for any sheaf $E$ and $\vec{x} \in \mathbf{L}(\mathbf{w})$.

W. Crawley-Boevey: Department of Pure Mathematics, University of Leeds, Leeds LS2 9JT, UK; e-mail: w.crawley-boevey@ leeds.ac.uk

Mathematics Subject Classification (2010): Primary 14H60, 16G20 
Every sheaf is the direct sum of a 'torsion-free' sheaf, which has a filtration by sheaves of the form $\mathcal{O}(\vec{x})$, and a finite-length sheaf, and the latter are easily described. There are simple sheaves $S_{a}\left(a \in \mathbb{P}^{1} \backslash D\right)$ and $S_{i j}\left(1 \leq i \leq k, 0 \leq j \leq w_{i}-1\right)$. They have

$$
\operatorname{dim} \operatorname{Hom}\left(\mathcal{O}(r \vec{c}), S_{i j}\right)=\boldsymbol{\delta}_{j 0}, \quad \operatorname{dim} \operatorname{Ext}^{1}\left(S_{i j}, \mathcal{O}(r \vec{c})\right)=\boldsymbol{\delta}_{j 1}
$$

where $\delta$ is the Kronecker delta function, and the only extensions between them are

$$
\operatorname{dim} \operatorname{Ext}^{1}\left(S_{a}, S_{a}\right)=1, \quad \operatorname{dim} \operatorname{Ext}^{1}\left(S_{i j}, S_{i \ell}\right)=1 \quad\left(\ell \equiv j-1\left(\bmod w_{i}\right)\right) .
$$

For each simple sheaf $S$ and $r>0$ there is a unique sheaf $S[r]$, with length $r$ and top $S$, which is uniserial, meaning that it has a unique composition series. These are all the finite-length indecomposable sheaves.

There is a root system associated to $\mathbf{w}$ via the graph

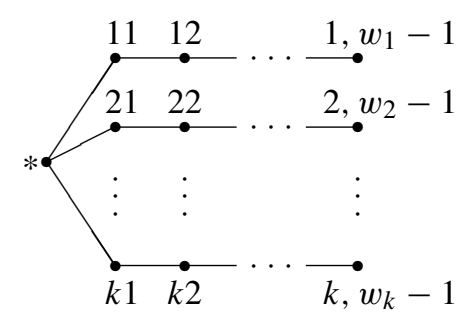

whose vertex set $I$ consists of $*$ and vertices denoted $i j$ or $i, j$ for $1 \leq i \leq k$ and $1 \leq j \leq w_{i}-1$. Let $\mathfrak{g}$ be the Kac-Moody Lie algebra (over $\mathbb{C}$ ) with generators $e_{v}, f_{v}, h_{v}$ $(v \in I)$ and relations

$$
\left\{\begin{array}{l}
{\left[h_{u}, h_{v}\right]=0, \quad\left[e_{u}, f_{v}\right]=\boldsymbol{\delta}_{u v} h_{v},} \\
{\left[h_{u}, e_{v}\right]=a_{u v} e_{v}, \quad\left[h_{u}, f_{v}\right]=-a_{u v} f_{v},} \\
\left(\operatorname{ad} e_{u}\right)^{1-a_{u v}}\left(e_{v}\right)=0, \quad\left(\operatorname{ad} f_{u}\right)^{1-a_{u v}}\left(f_{v}\right)=0 \quad(\text { if } u \neq v),
\end{array}\right.
$$

where the (symmetric) generalized Cartan matrix $\left(a_{u v}\right)$ has diagonal entries 2 and offdiagonal entries -1 if $u$ and $v$ are joined by an edge and otherwise 0 . The root lattice $\Gamma$ is the free additive group on symbols $\alpha_{v}(v \in I)$, and there is a symmetric bilinear form on it defined by $\left(\alpha_{u}, \alpha_{v}\right)=a_{u v}$. Now $\mathfrak{g}$ is graded by $\Gamma$, with $\operatorname{deg} e_{v}=\alpha_{v}, \operatorname{deg} f_{v}=-\alpha_{v}$ and $\operatorname{deg} h_{v}=0$, and the root system is $\Delta=\left\{0 \neq \alpha \in \Gamma \mid \mathfrak{g}_{\alpha} \neq 0\right\}$. Recall that there are real roots, obtained from the simple roots $\alpha_{v}$ by a sequence of reflections $s_{u}(\alpha)=$ $\alpha-\left(\alpha, \alpha_{u}\right) \alpha_{u}$, and there may also be imaginary roots.

The loop algebra of $\mathfrak{g}$ is $L \mathfrak{g}=\mathfrak{g}\left[t, t^{-1}\right]$, but more appropriate is an extension $\mathcal{L} \mathfrak{g}$ with generators $e_{v r}, f_{v r}, h_{v r}(v \in I, r \in \mathbb{Z})$ and $c$ subject to the relations

$$
\left\{\begin{array}{l}
c \text { central, } \quad\left[e_{v r}, e_{v s}\right]=0, \quad\left[f_{v r}, f_{v s}\right]=0, \\
{\left[h_{u r}, h_{v s}\right]=r a_{u v} \delta_{r+s, 0} c, \quad\left[e_{u r}, f_{v s}\right]=\delta_{u v}\left(h_{v, r+s}+r \boldsymbol{\delta}_{r+s, 0} c\right),} \\
{\left[h_{u r}, e_{v s}\right]=a_{u v} e_{v, r+s}, \quad\left[h_{u r}, f_{v s}\right]=-a_{u v} f_{v, r+s},} \\
\left(\operatorname{ad} e_{u 0}\right)^{1-a_{u v}}\left(e_{v s}\right)=0, \quad\left(\operatorname{ad} f_{u 0}\right)^{1-a_{u v}}\left(f_{v s}\right)=0 \quad(\text { if } u \neq v) ;
\end{array}\right.
$$


see [15] and [19, §1.3]. The root lattice for either algebra is $\hat{\Gamma}=\Gamma \oplus \mathbb{Z} \delta$ with deg $e_{v} t^{r}=$ $\operatorname{deg} e_{v r}=\alpha_{v}+r \delta, \operatorname{deg} f_{v} t^{r}=\operatorname{deg} f_{v r}=-\alpha_{v}+r \delta, \operatorname{deg} h_{v} t^{r}=\operatorname{deg} h_{v r}=r \delta$ and $\operatorname{deg} c=0$, and the set of roots for either algebra is

$$
\hat{\Delta}=\{\alpha+r \delta \mid \alpha \in \Delta, r \in \mathbb{Z}\} \cup\{r \delta \mid 0 \neq r \in \mathbb{Z}\} .
$$

The real roots are $\alpha+r \delta$ with $\alpha$ real. If $\mathfrak{g}$ is of finite type, then $\mathcal{L} \mathfrak{g}$ is the corresponding affine Lie algebra, and if $\mathfrak{g}$ is of affine type, then $\mathcal{L} \mathfrak{g}$ is a toroidal algebra.

The Grothendieck group $K_{0}(\operatorname{coh} \mathbb{X})$ was computed by Geigle and Lenzing, and following Schiffmann [19] it can be identified with $\hat{\Gamma}$, with

$$
[\mathcal{O}(r \vec{c})]=\alpha_{*}+r \delta, \quad\left[S_{a}\right]=\delta, \quad\left[S_{i j}\right]= \begin{cases}\alpha_{i j} & (j \neq 0), \\ \delta-\sum_{\ell=1}^{w_{i}-1} \alpha_{i \ell} & (j=0) .\end{cases}
$$

The type of a sheaf is the corresponding element of $\hat{\Gamma}$. The symmetric bilinear form $(-,-)$ on $\Gamma$ extends to $\hat{\Gamma}$ by defining $(\delta,-)=0$, and it corresponds to the symmetrization of the Euler form

$$
\langle[X],[Y]\rangle=\operatorname{dim} \operatorname{Hom}(X, Y)-\operatorname{dim} \operatorname{Ext}^{1}(X, Y)
$$

on $K_{0}(\operatorname{coh} \mathbb{X})$. Now $K_{0}(\operatorname{coh} \mathbb{X})$ is partially ordered, with the positive cone being the classes of objects in coh $\mathbb{X}$. By (2) the corresponding partial ordering on $\hat{\Gamma}$ has as positive cone $\hat{\Gamma}_{+}$the non-negative linear combinations of the elements $\alpha_{*}+r \delta(r \in \mathbb{Z}), \delta$, $\alpha_{i j}$ and $\delta-\sum_{\ell=1}^{w_{i}-1} \alpha_{i \ell}$. Clearly every root is positive or negative.

Theorem 1. If $\mathbb{X}$ is a weighted projective line and $\phi \in \hat{\Gamma}$, there is an indecomposable sheaf in coh $\mathbb{X}$ of type $\phi$ if and only if $\phi$ is a positive root. There is a unique indecomposable for a real root, infinitely many for an imaginary root.

This is an analogue of Kac's Theorem [7, 8, 10] which describes the possible dimension vectors of indecomposable representations of quivers.

We remark that there is a complete classification of indecomposables if $\mathfrak{g}$ is of finite type [4], and also if $\mathfrak{g}$ is of affine type [12]. The latter is essentially equivalent to Ringel's classification [18] of representations of tubular algebras.

Lenzing [11, §4.2] showed that the category of torsion-free sheaves on $\mathbb{X}$ is equivalent to the category of (quasi) parabolic bundles on $\mathbb{P}^{1}$ of weight type $(D, \mathbf{w})$, that is, vector bundles $\pi: E \rightarrow \mathbb{P}^{1}$ equipped with a flag of subspaces

$$
\pi^{-1}\left(a_{i}\right) \supseteq E_{i 1} \supseteq \cdots \supseteq E_{i, w_{i}-1}
$$

for each $i$. This equivalence is not unique, but it can be chosen so that if $E$ is a parabolic bundle, then $[E]=\underline{\operatorname{dim}} E+(\operatorname{deg} E) \delta$. Here the dimension vector of $E$ is

$$
\underline{\operatorname{dim}} E=n_{*} \alpha_{*}+\sum_{i=1}^{k} \sum_{j=1}^{w_{i}-1} n_{i j} \alpha_{i j} \in \Gamma,
$$

with $n_{*}=\operatorname{rank} E$ and $n_{i j}=\operatorname{dim} E_{i j}$. Observe that the dimension vector is necessarily strict, meaning that $n_{*} \geq n_{i 1} \geq n_{i 2} \geq \cdots \geq n_{i, w_{i}-1} \geq 0$. We can now restate Theorem 1 as follows. 
Corollary. For each $d \in \mathbb{Z}$ there is an indecomposable parabolic bundle of dimension vector $\alpha \in \Gamma$ and degree $d$ if and only if $\alpha$ is a strict root for $\mathfrak{g}$. There is a unique indecomposable for a real root, and infinitely many for an imaginary root.

In [3] this result is shown to be related to the existence of matrices in prescribed conjugacy class closures with product equal to the identity. Using that, in case the matrices have generic eigenvalues, we gave a partial proof over the complex field.

Our proof of Theorem 1 uses Hall algebras. First we need a lemma, which we have observed with C. Geiß. Given a parabolic bundle $E$, the underlying vector bundle on $\mathbb{P}^{1}$ decomposes as a direct sum of line bundles of degrees $n_{1} \leq \cdots \leq n_{r}$. One might call $n_{r}-n_{1}$ the width of $E$.

Lemma 1. For any $\phi \in \hat{\Gamma}$ there is a bound, depending only on $\mathbf{w}$ and $\phi$, of the width of indecomposable parabolic bundles of type $\phi$.

Equivalently, for any $\phi, \psi \in \hat{\Gamma}$ there is a bound on $\operatorname{dim} \operatorname{Hom}(X, Y)$ (and so also on $\left.\operatorname{dim} \operatorname{Ext}^{1}(X, Y)\right)$ for $X, Y$ indecomposable of types $\phi, \psi$.

Proof. The argument is the same as for [1, Theorem 1]. Any torsion-free sheaf $E$ has a splitting by rank-one torsion-free sheaves $\left(L_{1}, \ldots, L_{r}\right)$, meaning that there is a chain $0=E_{0} \subset E_{1} \subset \cdots \subset E_{r}=E$ and $L_{i}=E_{i} / E_{i-1}$. The degree is defined for weighted projective lines by [4, Proposition 2.8], and using it one may consider splittings which are maximal in the sense that $L_{1}$ has maximal possible degree, and amongst these $L_{2}$ has maximal possible degree, etc.

By [4, Corollary 1.8.1] and the structure of the ring $S(\mathbf{w}, D)$, it is clear that there is an integer $h$ with $\operatorname{Hom}\left(L, L^{\prime}\right) \neq 0$ for any rank one torsion-free sheaves $L, L^{\prime}$ with $\operatorname{deg} L^{\prime}-\operatorname{deg} L>h$. If $\left(L_{1}, L_{2}\right)$ is a maximal splitting of $E$, then there there is an exact sequence

$$
\operatorname{Hom}\left(L_{1}(\vec{c}), E\right) \rightarrow \operatorname{Hom}\left(L_{1}(\vec{c}), L_{2}\right) \rightarrow \operatorname{Ext}^{1}\left(L_{1}(\vec{c}), L_{1}\right) .
$$

The right hand space is zero since $\operatorname{Ext}^{1}(\mathcal{O}(\vec{c}), \mathcal{O})=0$. If $\operatorname{deg} L_{2}-\operatorname{deg} L_{1}(\vec{c})>h$, then the middle space is nonzero, and so $\operatorname{Hom}\left(L_{1}(\vec{c}), E\right) \neq 0$. Taking the image of such a map, and enlarging it so that the quotient of $E$ by this subsheaf is torsion-free, one contradicts the maximality of the splitting. Thus we must have $\operatorname{deg} L_{2}-\operatorname{deg} L_{1}(\vec{c}) \leq h$, giving a bound of the form $\operatorname{deg} L_{2}-\operatorname{deg} L_{1} \leq h^{\prime}$, for some $h^{\prime}$. As in [1, Lemma 4] this gives bounds $\operatorname{deg} L_{i}-\operatorname{deg} L_{i-1} \leq h^{\prime}$ for any maximal splitting $\left(L_{1}, \ldots, L_{r}\right)$, so $\operatorname{deg} L_{i} \leq \operatorname{deg} L_{1}+(i-1) h^{\prime}$.

Now suppose $E$ is indecomposable, and let $\left(L_{1}, \ldots, L_{r}\right)$ be a maximal splitting. We show by induction that $\operatorname{deg} L_{i} \geq \operatorname{deg} L_{1}-(i-1) h^{\prime \prime}$ where $h^{\prime \prime}=\delta(\vec{\omega})$ in the notation of [4]. For $1<i \leq n$, since $E$ is indecomposable we must have $\operatorname{Ext}^{1}\left(E / E_{i-1}, E_{i-1}\right) \neq 0$, so $\operatorname{Hom}\left(E_{i-1},\left(E / E_{i-1}\right)(\vec{\omega})\right) \neq 0$ by Serre duality, and hence $\operatorname{Hom}\left(L_{j}(-\vec{\omega}),\left(E / E_{i-1}\right)\right)$ $\neq 0$ for some $j<i$. This implies that $E / E_{i-1}$ has a subsheaf of degree at least deg $L_{j}-h^{\prime \prime}$, so by maximality $\operatorname{deg} L_{i} \geq \operatorname{deg} L_{j}-h^{\prime \prime} \geq \operatorname{deg} L_{1}-(i-1) h^{\prime \prime}$ by induction.

The assertion follows.

For an alternative approach see [13, Theorem 2.9]. 


\section{Hall algebras}

Let $K$ be a finite field and let $\mathcal{R}$ be a triangulated $K$-category which is 2-periodic, meaning that the shift functor $T$ satisfies $T^{2}=1$. There is a bilinear form on $K_{0}(\mathcal{R})$,

$$
\langle[X],[Y]\rangle=\operatorname{dim} \operatorname{Hom}(X, Y)-\operatorname{dim} \operatorname{Hom}(X, T Y),
$$

and let $(-,-)$ be its symmetrization. Let ind $\mathcal{R}$ be a set of representatives of the isomorphism classes of indecomposable objects in $\mathcal{R}$. Assume that $\mathcal{R}$ is finitary, meaning that it has finite Hom spaces and $\{X \in$ ind $\mathcal{R} \mid[X]=\phi\}$ is finite for all $\phi \in K_{0}(\mathcal{R})$. For $X \in \operatorname{ind} \mathcal{R}$, define $d(X)=\operatorname{dim}(\operatorname{End}(X) / \operatorname{rad} \operatorname{End}(X))$, and assume for simplicity that $K_{0}(\mathcal{R})$ is torsion-free, generated by indecomposables with $d(X)=1$, and that $[X]$ is divisible in $K_{0}(X)$ by $d(X)$ for all $X \in$ ind $\mathcal{R}$. Define

$$
F_{X Y}^{Z}=\mid\{\text { triangles } Y \rightarrow Z \rightarrow X \rightarrow\} / \operatorname{Aut}(X) \times \operatorname{Aut}(Y) \mid \text {. }
$$

Let $\Lambda$ be a commutative ring. Assuming that $|K|=1$ in $\Lambda$, Peng and Xiao [17, 6] proved that

$$
L_{\Lambda}(\mathcal{R})=\left(\Lambda \otimes_{\mathbb{Z}} K_{0}(\mathcal{R})\right) \oplus \bigoplus_{X \in \text { ind } \mathcal{R}} \Lambda u_{X}
$$

becomes a Lie algebra over $\Lambda$ with bracket

$$
\left[u_{X}, u_{Y}\right]= \begin{cases}\sum_{Z \in \operatorname{ind} \mathcal{R}}\left(F_{X Y}^{Z}-F_{Y X}^{Z}\right) u_{Z} & (X \supsetneqq T Y), \\ 1 \otimes \frac{[X]}{d(X)} & (X \cong T Y),\end{cases}
$$

and $\left[1 \otimes \phi, u_{X}\right]=-(\phi,[X]) u_{X}$ and $[1 \otimes \phi, 1 \otimes \psi]=0$ for $\phi, \psi \in K_{0}(\mathcal{R})$.

We now consider weighted projective lines over finite fields, in the case when the marked points are all defined over the finite field. The category $\operatorname{coh} \mathbb{X}$ is still defined and well-behaved (see [11] or [19]). Schiffmann [19] has considered its Hall algebra, and related it to a quantum group for the positive part of $\mathcal{L} \mathfrak{g}$. To apply the construction of Peng and Xiao one uses the quotient category

$$
\mathcal{R}_{\mathbb{X}}=D^{b}(\operatorname{coh} \mathbb{X}) /\left(T^{2}\right),
$$

called the root category, whose objects are representatives of the orbits of $T^{2}$ on $D^{b}(\operatorname{coh} \mathbb{X})$, and with

$$
\operatorname{Hom}_{\mathcal{R}_{\mathbb{X}}}(X, Y)=\bigoplus_{n \in \mathbb{Z}} \operatorname{Hom}_{D^{b}(\operatorname{coh} \mathbb{X})}\left(X, T^{2 n} Y\right) .
$$

This is known to be a 2-periodic triangulated category by [16, Lemma 2.3]. (See also [14, $\S 3]$ for the transition from hereditary algebras to hereditary abelian categories.) Since $\operatorname{coh} \mathbb{X}$ is hereditary, the indecomposable objects in $D^{b}(\operatorname{coh} \mathbb{X})$ are the shifts of the indecomposables in coh $\mathbb{X}$, and hence

$$
\text { ind } \mathcal{R}_{\mathbb{X}}=(\text { ind } \operatorname{coh} \mathbb{X}) \cup\{T Y \mid Y \in \text { ind } \operatorname{coh} \mathbb{X}\} \text {. }
$$


Recall that any triangle $X \rightarrow Y \rightarrow Z \rightarrow$ can be rotated to give a triangle $Y \rightarrow Z \rightarrow$ $T X \rightarrow$. Any triangle $X \rightarrow Y \rightarrow Z \rightarrow$ in $\mathcal{R}_{\mathbb{X}}$ with $X, Y, Z$ indecomposable can be rotated sufficiently so that $X$ and $Z$ are in coh $\mathbb{X}$, and in this case $Y$ must also be, and then such triangles are in 1-1 correspondence with short exact sequences $0 \rightarrow X \rightarrow Y \rightarrow$ $Z \rightarrow 0$.

Assuming that the base field $K$ is finite and $|K|=1$ in $\Lambda$, the construction of Peng and Xiao gives a Lie algebra $L_{\Lambda}\left(\mathcal{R}_{\mathbb{X}}\right)$ with triangular decomposition

$$
L_{\Lambda}\left(\mathcal{R}_{\mathbb{X}}\right)=\left(\bigoplus_{X \in \text { ind coh } \mathbb{X}} \Lambda u_{X}\right) \oplus\left(\Lambda \otimes_{\mathbb{Z}} \hat{\Gamma}\right) \oplus\left(\bigoplus_{Y \in \text { ind coh } \mathbb{X}} \Lambda u_{T Y}\right)
$$

We define $b_{X}$ for $X \in$ ind $\mathcal{R}_{\mathbb{X}}$ by $b_{Y}=u_{Y}$ and $b_{T Y}=-u_{T Y}$ for $Y \in$ ind $\operatorname{coh} \mathbb{X}$. If $S$ is a simple sheaf, we extend the notation $S[r]$ to $r<0$ by defining $S[r]=T Y$, where $Y$ is the unique uniserial sheaf of length $-r$ with $\operatorname{Ext}^{1}(Y, S) \neq 0$, so that $\operatorname{Hom}(S[r], S) \neq 0$. Let $H_{r}$ be the set of $X \in$ ind $\mathcal{R}_{\mathbb{X}}$ of type $r \delta$ and with $\operatorname{Hom}\left(X, S_{i j}\right)=0$ for all $1 \leq i \leq k$, $1 \leq j \leq w_{i}-1$, and set $\mathbf{h}_{r}=\sum_{X \in H_{r}} d(X) b_{X}$.

Theorem 2. The following elements of $L_{\Lambda}\left(\mathcal{R}_{\mathbb{X}}\right)$ satisfy the relations (1) for $\mathcal{L} \mathfrak{g}:$

$$
\begin{gathered}
e_{v r}=\left\{\begin{array}{ll}
b_{S_{i j}\left[r w_{i}+1\right]} & (v=i j), \\
b_{\mathcal{O}(r \vec{c})} & (v=*),
\end{array} \quad f_{v r}= \begin{cases}b_{S_{i, j-1}\left[r w_{i}-1\right]} & (v=i j), \\
b_{T \mathcal{O}(-r \vec{c})} & (v=*),\end{cases} \right. \\
c=-1 \otimes \delta, \quad h_{v r}= \begin{cases}-1 \otimes \alpha_{v} & (r=0), \\
b_{S_{i j}\left[r w_{i}\right]}-b_{S_{i, j-1}\left[r w_{i}\right]} & (r \neq 0, v=i j), \\
\mathbf{h}_{r} & (r \neq 0, v=*) .\end{cases}
\end{gathered}
$$

See also [14], where elliptic Lie algebra generators are found in $L_{\Lambda}\left(\mathcal{R}_{\mathbb{X}}\right)$ for $\mathfrak{g}$ of affine type, [19], where the Hall algebra of coh $\mathbb{X}$ is considered, and [9], where doubled Hall algebras are considered.

\section{Proof of Theorem 2}

Lemma 2. If $0 \rightarrow X \rightarrow Y \rightarrow Z \rightarrow 0$ is a short exact sequence of indecomposable finite-length sheaves, then up to automorphisms of any two of $X, Y, Z$, any other exact sequence with the same terms is equivalent to this one.

Proof. Since $Y$ is uniserial, it has a unique subsheaf $Y^{\prime}$ isomorphic to $X$, from which it is clear that there is a unique sequence up to the action of $\operatorname{Aut}(X) \times \operatorname{Aut}(Z)$. For the action of $\operatorname{Aut}(X) \times \operatorname{Aut}(Y)$, say, we reduce to the case where $X, Y, Z$ are finite-dimensional modules for a finite-dimensional serial algebra, and we may assume that $Y$ is projective. Then any two epimorphisms $Y \rightarrow Z$ are equivalent via an element of $\operatorname{Aut}(Y)$, and the result follows.

Lemma 3. $T S_{i j}[r]=S_{i, j-r}[-r]$ where the subscript $j-r$ is computed modulo $w_{i}$.

Proof. Clear. 
Lemma 4. One has

$$
\left[b_{S_{i j}[r]}, b_{S_{i k}[s]}\right]= \begin{cases}\boldsymbol{\delta}_{j-r, k} b_{S_{i j}[r+s]}-\boldsymbol{\delta}_{j, k-s} b_{S_{i k}[r+s]} & (r+s \neq 0), \\ -\boldsymbol{\delta}_{j-r, k} \otimes\left[S_{i j}[r]\right] & (r+s=0),\end{cases}
$$

where the subscripts $j-r$ and $k-s$ are computed modulo $w_{i}$.

Proof. If $r, s>0$, then one gets a positive contribution of $u_{X}$ for short exact sequences $0 \rightarrow S_{i k}[s] \rightarrow X \rightarrow S_{i j}[r] \rightarrow 0$, and a negative contribution for short exact sequences $0 \rightarrow S_{i j}[r] \rightarrow X \rightarrow S_{i k}[s] \rightarrow 0$. The condition for the existence of nonsplit sequences is given by the $\delta$ 's. In each case there is a unique possible middle term, and the coefficient is 1 by Lemma2

If $r, s<0$ the argument is similar.

If $r>0, s<0$, one gets a contribution of $u_{X}$ for $X$ in a triangle $S_{i k}[s] \rightarrow X \rightarrow$ $S_{i j}[r] \rightarrow$ or $S_{i j}[r] \rightarrow X \rightarrow S_{i k}[s] \rightarrow$. Rotating, these become triangles $X \rightarrow S_{i j}[r] \rightarrow$ $S_{i, k-s}[-s] \rightarrow$ and $S_{i, k-s}[-s] \rightarrow S_{i j}[r] \rightarrow X \rightarrow$. Suppose that $r \geq-s$ (the reverse is similar). Then $X$ must be a sheaf in both cases, corresponding to short exact sequences $0 \rightarrow X \rightarrow S_{i j}[r] \rightarrow S_{i, k-s}[-s] \rightarrow 0$ and $0 \rightarrow S_{i, k-s}[-s] \rightarrow S_{i j}[r] \rightarrow X \rightarrow 0$. The existence of such sequences is given by the $\delta$ 's, and in each case there is a unique possible $X$.

Lemma 5. There is a short exact sequence $0 \rightarrow \mathcal{O}(r \vec{c}) \rightarrow X \rightarrow S_{i j}[s] \rightarrow 0$ with $X$ indecomposable if and only if $j \equiv s\left(\bmod w_{i}\right)$, and then $X \cong \mathcal{O}\left(r \vec{c}+s \vec{x}_{i}\right)$.

Proof. If $X$ is indecomposable it is of the form $\mathcal{O}(\vec{x})$ for some $\vec{x}$, and by considering the type, one must have $\vec{x}=r \vec{c}+s \vec{x}_{i}$. Now since there is a nonzero homomorphism $\mathcal{O}(\vec{x}) \rightarrow S_{i j}$, one has $j \equiv s\left(\bmod w_{i}\right)$.

Lemma 6. If $X, Y \in$ ind $\mathcal{R}_{\mathbb{X}}$ and $[X]=r \delta,[Y]=s \delta$ then $\left[b_{X}, b_{Y}\right]=0$ if $X \supsetneqq T Y$.

Proof. To have any chance of $\left[b_{X}, b_{Y}\right]$ being nonzero, the simple sheaves involved in $X$ and $Y$ must all be of the form $S_{a}$ or must all be of the form $S_{i j}$ for fixed $i$. The latter case follows from Lemma 4 . The former case is analogous.

Lemma 7. $H_{-r}=\left\{T Y \mid Y \in H_{r}\right\}$.

Proof. Clear.

Lemma 8. $\sum_{X \in H_{r}} d(X)=2$ in $\Lambda$.

Proof. We may assume that $r>0$. The restriction $\operatorname{Hom}\left(X, S_{i j}\right)=0$ for all $1 \leq i \leq k$, $1 \leq j \leq w_{i}-1$, ensures that the marked points can each contribute at most one indecomposable. Thus this is a question about torsion sheaves on $\mathbb{P}^{1}$. The point at infinity contributes one indecomposable sheaf, and the rest correspond to indecomposable $r$-dimensional modules for the polynomial ring $K[x]$. Now absolutely indecomposable modules are given by Jordan blocks, so the number is equal to the size of the field, and as this is equal to 1 in $\Lambda$, formula $(\alpha)$ on page 91 of [8] gives the result.

We now verify that the elements of Theorem 2 satisfy the relations $(1)$ for $\mathcal{L} \mathfrak{g}$. The arguments are all standard in the theory of Hall algebras. 
(i) $c$ central. This is clear since $(\delta,-)=0$.

(ii) $\left[e_{v r}, e_{v s}\right]=0$.

(a) If $v=i j$ this follows from Lemma 4

(b) If $v=*$ we want $\left[u_{\mathcal{O}(r \vec{c})}, u_{\mathcal{O}(s \vec{c})}\right]=0$. The sheaves $\mathcal{O}(r \vec{c})$ all lie in a subcategory of $\operatorname{coh} \mathbb{X}$ which is equivalent to $\operatorname{coh} \mathbb{P}^{1}$. In any extension, the middle term lives in this category coh $\mathbb{P}^{1}$, but here the indecomposables are all line bundles.

(iii) $\left[f_{v r}, f_{v s}\right]=0$. Similar to (ii).

(iv) $\left[h_{u r}, h_{v s}\right]=r a_{u v} \boldsymbol{\delta}_{r+s, 0} c$. Expanding the left hand side, observe that every $u_{X}$ which occurs has $[X]=r \delta$ or $s \delta$, so in the radical of the symmetric bilinear form. Thus by Lemma 6, the only way to not get zero is if $h_{u r}$ involves a $u_{X}$ and $h_{v s}$ involves the corresponding $u_{T X}$. Thus the only possibilities are $\left[h_{u r}, h_{v,-r}\right]$ with $r \neq 0$. By symmetry we may assume that $r>0$.

(a) By Lemmas 7 and 8 we have

$$
\begin{aligned}
{\left[h_{*, r}, h_{*,-r}\right] } & =\sum_{X, Y \in H_{r}} d(X) d(Y)\left[b_{X}, b_{T Y}\right]=-\sum_{X \in H_{r}} d(X)^{2}\left[u_{X}, u_{T X}\right] \\
& =-\sum_{X \in H_{r}} d(X)^{2} 1 \otimes[X] / d(X)=-1 \otimes \sum_{X \in H_{r}} d(X)[X] \\
& =-1 \otimes r \delta \sum_{X \in H_{r}} d(X)=2 r(-1 \otimes \delta)=2 r c .
\end{aligned}
$$

(b) $\left[h_{i j, r}, h_{i j,-r}\right]=\left[b_{S_{i j}\left[r w_{i}\right]}-b_{S_{i, j-1}\left[r w_{i}\right]}, b_{S_{i j}\left[-r w_{i}\right]}-b_{S_{i, j-1}\left[-r w_{i}\right]}\right]$. Expanding this, the cross terms vanish by the argument above, giving

$$
\left[b_{S_{i j}\left[r w_{i}\right]}, b_{S_{i j}\left[-r w_{i}\right]}\right]+\left[b_{S_{i, j-1}\left[r w_{i}\right]}, b_{S_{i, j-1}\left[-r w_{i}\right]}\right]=-2 \otimes r \delta=2 r c .
$$

(c) $\left[h_{i j, r}, h_{k \ell,-r}\right]$ can only be nonzero, by the argument above, if $k=i$ and $\ell=j$ or $j \pm 1$. If $\ell=j \pm 1$, then one gets a cross term, so the result is $-r c$.

(d) For $\left[h_{*, r}, h_{i j,-r}\right]$, the only nonzero term which might occur comes from $S_{i 0}\left[r w_{i}\right] \in H_{r}$, giving $\left[b_{S_{i 0}\left[r w_{i}\right]},-b_{S_{i 0}\left[-r w_{i}\right]}\right]$ provided that $j=1$. This gives $-r c$.

(v) $\left[e_{u r}, f_{v s}\right]=\boldsymbol{\delta}_{u v}\left(h_{v, r+s}+r \boldsymbol{\delta}_{r+s, 0} c\right)$.

(a) For $\left[e_{i j, r}, f_{k \ell, s}\right]$, if $r+s=0$ then

$$
\begin{aligned}
{\left[e_{i j, r}, f_{k \ell, s}\right] } & =\left[b_{S_{i j}\left[r w_{i}+1\right]}, b_{S_{k, \ell-1}\left[s w_{k}-1\right]}\right] \\
& =-\boldsymbol{\delta}_{i k} \boldsymbol{\delta}_{j-\left(r w_{i}+1\right), \ell-1} \otimes\left[S_{i j}\left[r w_{i}+1\right]\right] \\
& =-\boldsymbol{\delta}_{i k} \boldsymbol{\delta}_{j \ell} \otimes\left(\alpha_{i j}+r \delta\right)=\boldsymbol{\delta}_{i k} \boldsymbol{\delta}_{j \ell}\left(h_{i j, 0}+r c\right),
\end{aligned}
$$

and if $r+s \neq 0$ then

$$
\begin{aligned}
{\left[e_{i j, r}, f_{k \ell, s}\right] } & =\left[b_{S_{i j}\left[r w_{i}+1\right]}, b_{S_{k, \ell-1}\left[s w_{k}-1\right]}\right] \\
& =\boldsymbol{\delta}_{i k} \boldsymbol{\delta}_{j \ell}\left(b_{S_{i j}\left[(r+s) w_{i}\right]}-b_{S_{i, j-1}\left[(r+s) w_{i}\right]}\right) \\
& =\boldsymbol{\delta}_{i k} \boldsymbol{\delta}_{j \ell} h_{i j, r+s} .
\end{aligned}
$$


(b) For $\left[e_{*, r}, f_{*, s}\right]$, if $r+s=0$ then

$$
\begin{aligned}
{\left[e_{*, r}, f_{*, s}\right] } & =-\left[u_{\mathcal{O}(r \vec{c})}, u_{T \mathcal{O}(-s \vec{c})}\right]=-1 \otimes[\mathcal{O}(r \vec{c})] \\
& =-1 \otimes\left(\alpha_{*}+r \delta\right)=h_{*, 0}+r c
\end{aligned}
$$

so suppose that $r+s \neq 0$. In computing $\left[e_{*, r}, f_{*, s}\right]=-\left[u_{\mathcal{O}(r \vec{c})}, u_{T \mathcal{O}(-s \vec{c})}\right]$, one gets a negative contribution of $u_{X}$ for triangles $T \mathcal{O}(-s \vec{c}) \rightarrow X \rightarrow$ $\mathcal{O}(r \vec{c}) \rightarrow$, which is only possible when $X=T Y$ with $Y$ a uniserial sheaf, and a positive contribution for triangles $\mathcal{O}(r \vec{c}) \rightarrow X \rightarrow T \mathcal{O}(-s \vec{c}) \rightarrow$, which is possible for $X=Y$, a uniserial sheaf. Thus one gets a positive contribution of $b_{X}$ in each case. In computing the coefficients, one may apply a shift to the triangles, so one sees that the answer only depends on $r, s$ through their sum $t=r+s$. Thus one gets contributions for exact sequences $0 \rightarrow \mathcal{O}(t \vec{c}) \rightarrow \mathcal{O} \rightarrow Y \rightarrow 0$ and $0 \rightarrow \mathcal{O}(-t \vec{c}) \rightarrow \mathcal{O} \rightarrow Y \rightarrow 0$. Assuming that $t>0$ (the case $t<0$ is similar), only the latter are involved. The possible $Y$ are those in $H_{t}$, and for such $Y$, if $S$ is the simple in its top, and $d=d(Y)=d(S)$, then there are $t / d$ copies of $S$ involved in $Y$. Now $\operatorname{Hom}(\mathcal{O}, Y)$ has dimension $t$, and the nonepimorphisms give a subspace of dimension $t-d$. Thus the number of exact sequences is

$$
(q-1)\left(q^{t}-q^{t-d}\right) .
$$

Factoring out by the automorphisms of $\mathcal{O}(-t \vec{c})$ and $\mathcal{O}$, which act freely, one gets

$$
\frac{q^{t}-q^{t-d}}{q-1}=q^{t-d} \frac{q^{d}-1}{q-1} .
$$

In $\Lambda$ this is $d$, so $\sum_{Y \in H_{t}} d(Y) b_{Y}=\mathbf{h}_{t}=h_{*, t}$.

(c) For $\left[e_{*, r}, f_{i j, s}\right]$, one gets contributions from triangles $S_{i, j-1}\left[s w_{i}-1\right] \rightarrow X \rightarrow$ $\mathcal{O}(r \vec{c}) \rightarrow$ and $\mathcal{O}(r \vec{c}) \rightarrow X \rightarrow S_{i, j-1}\left[s w_{i}-1\right] \rightarrow$. Rotating, the first becomes $X \rightarrow \mathcal{O}(r \vec{c}) \rightarrow S_{i j}\left[-s w_{i}+1\right] \rightarrow$ by Lemma 3 Now there can be nonzero homomorphisms from $\mathcal{O}(r \vec{c})$ to $S_{i j}\left[-s w_{i}+1\right]$ only if the latter is a sheaf, but then there are no epimorphisms since $j \neq 0$. The second becomes $X \rightarrow$ $S_{i, j-1}\left[s w_{i}-1\right] \rightarrow T \mathcal{O}(r \vec{c}) \rightarrow$ and there can only be nonzero homomorphisms from $S_{i, j-1}\left[s w_{i}-1\right]$ to $T \mathcal{O}(r \vec{c})$ if $S_{i, j-1}\left[s w_{i}-1\right]$ is a sheaf. Thus one deals with short exact sequences $0 \rightarrow \mathcal{O}(r \vec{c}) \rightarrow X \rightarrow S_{i, j-1}\left[s w_{i}-1\right] \rightarrow 0$. Since $X$ is indecomposable, it must be a torsion-free sheaf. Now if $f$ is the morphism $X \rightarrow S_{i, j-1}\left[s w_{i}-1\right]$ and $S$ is the socle of $S_{i, j-1}\left[s w_{i}-1\right]$, then $f^{-1}(S)$ must also be torsion-free. But the sequence $0 \rightarrow \mathcal{O}(r \vec{c}) \rightarrow f^{-1}(S) \rightarrow S \rightarrow 0$ splits since $S \cong S_{i, j+1}$.

(d) $\left[e_{i j, r}, f_{*, s}\right]$ is similar to (c).

(vi) $\left[h_{u r}, e_{v s}\right]=a_{u v} e_{v, r+s}$. If $r=0$ then

$$
\left[h_{u r}, e_{v s}\right]=\left[-1 \otimes \alpha_{u}, e_{v s}\right]=\left(\alpha_{u}, \alpha_{v}+s \delta\right) e_{v s}
$$

as required, so suppose $r \neq 0$. We assume that $r>0$. (The case $r<0$ is similar.) 
(a) $\left[h_{i j, r}, e_{k \ell, s}\right]=\left[b_{S_{i j}\left[r w_{i}\right]}-b_{S_{i, j-1}\left[r w_{i}\right]}, b_{S_{k \ell}\left[s w_{k}+1\right]}\right]$, and Lemma 4 gives the result.

(b) $\left[h_{i j, r}, e_{*, s}\right]=\left[b_{S_{i j}\left[r w_{i}\right]}-b_{S_{i, j-1}\left[r w_{i}\right]}, b_{\mathcal{O}(s \vec{c})}\right]$. In expanding, one gets contributions $u_{X}$ only for short exact sequences with middle term $X$ and end terms the sheaves in the expression. By the argument in (v)(c), the only possible extension with indecomposable middle term is $0 \rightarrow \mathcal{O}(s \vec{c}) \rightarrow X \rightarrow S_{i 0}\left[r w_{i}\right] \rightarrow 0$, and then $X \cong \mathcal{O}((r+s) \vec{c})$. There is only one such extension, modulo automorphisms, giving $\left[h_{i j, r}, e_{*, s}\right]=-b_{\mathcal{O}((r+s) \vec{c})}=-e_{*, r+s}$.

(c) $\left[h_{*, r}, e_{i j, s}\right]=\sum_{X \in H_{r}} d(X)\left[b_{X}, b_{S_{i j}\left[s w_{i}+1\right]}\right]$. One gets a contribution of $u_{Y}$ for triangles $S_{i j}\left[s w_{i}+1\right] \rightarrow Y \rightarrow X \rightarrow$ and $X \rightarrow Y \rightarrow S_{i j}\left[s w_{i}+1\right] \rightarrow$.

If $s \geq 0$ these correspond to short exact sequences $0 \rightarrow S_{i j}\left[s w_{i}+1\right] \rightarrow$ $Y \rightarrow X \rightarrow 0$ and $0 \rightarrow X \rightarrow Y \rightarrow S_{i j}\left[s w_{i}+1\right] \rightarrow 0$. For the first, there are no indecomposable $Y$, and for the second there is only an exact sequence with $Y$ indecomposable if $j=1$ and $X \cong S_{i 0}\left[r w_{i}\right]$, and then $\left[h_{*, r}, e_{i j, s}\right]=$ $-u_{S_{i j}\left[r w_{i}+s w_{i}+1\right]}=-e_{i j, r+s}$.

If $s<0$ and $r+s \geq 0$, the triangles correspond to short exact sequences $0 \rightarrow Y \rightarrow X \rightarrow S_{i, j-1}\left[-s w_{i}-1\right] \rightarrow 0$ and $0 \rightarrow S_{i, j-1}\left[-s w_{i}-1\right] \rightarrow X \rightarrow$ $Y \rightarrow 0$. and the only possibility is $j=1$ and $Y \cong S_{i, w_{i}-1}\left[r w_{i}+s w_{i}+1\right]$ in the first of these, so again $\left[h_{*, r}, e_{i j, s}\right]=-u_{S_{i j}\left[r w_{i}+s w_{i}+1\right]}=-e_{i j, r+s}$.

If $r+s<0$, the triangles correspond to short exact sequences $0 \rightarrow X \rightarrow$ $S_{i, j-1}\left[-s w_{i}-1\right] \rightarrow T Y \rightarrow 0$ and $0 \rightarrow T Y \rightarrow S_{i, j-1}\left[-s w_{i}-1\right] \rightarrow X \rightarrow 0$, and the only possibility is $j=1$ and $T Y \cong S_{i 0}\left[-r w_{i}-s w_{i}-1\right]$, and again $\left[h_{*, r}, e_{i j, s}\right]=-e_{i j, r+s}$.

(d) $\left[h_{*, r}, e_{*, s}\right]=\sum_{X \in H_{r}} d(X)\left[b_{X}, b_{\mathcal{O}(s \vec{c})}\right]$. Computing the brackets on the right hand side, one gets a positive contribution of $u_{Y}$ for triangles $\mathcal{O}(s \vec{c}) \rightarrow Y \rightarrow$ $X \rightarrow$, and a negative contribution for triangles $X \rightarrow Y \rightarrow \mathcal{O}(s \vec{c}) \rightarrow$. In the first case $Y$ must be a sheaf. In the second it must also be a sheaf, but there are no nonsplit extensions. Consider exact sequences $0 \rightarrow \mathcal{O}(s \vec{c}) \rightarrow Y \rightarrow$ $X \rightarrow 0$. The only possible $Y$ is $\mathcal{O}((r+s) \vec{c})$, and the number of sequences modulo automorphisms of $\mathcal{O}(s \vec{c})$ and $X$ is 1 . Thus $\sum_{X \in H_{r}} d(X) u_{\mathcal{O}((r+s) \vec{c})}=$ $2 e_{*, r+s}$ by Lemma 8

(vii) $\left[h_{u r}, f_{v s}\right]=-a_{u v} f_{v, r+s}$. Similar to (vi).

(viii) $\left(\operatorname{ad} e_{u 0}\right)^{1-a_{u v}}\left(e_{v s}\right)=0$ for $u \neq v$.

(a) $\left[e_{i j, 0}, e_{k \ell, s}\right]=0$ for $k \neq i$ or $\ell \neq j \pm 1$ by Lemma 4 .

(b) $\left[e_{*, 0}, e_{i j, s}\right]=0$ for $j>1$. One gets a contribution of $u_{X}$ for sheaves belonging to short exact sequences $0 \rightarrow \mathcal{O} \rightarrow X \rightarrow S_{i j}\left[s w_{i}+1\right] \rightarrow 0$. Now the epimorphism $S_{i j}\left[s w_{i}+1\right] \rightarrow S_{i j}$ induces an epimorphism $X \rightarrow S_{i j}$. If $L$ is its kernel, then $L$ is an extension of $\mathcal{O}$ by $S_{i, j-1}\left[s w_{i}\right]$, so $L \cong \mathcal{O}(s \vec{c})$. But there is no nonsplit extension $0 \rightarrow \mathcal{O}(s \vec{c}) \rightarrow X \rightarrow S_{i j} \rightarrow 0$ for $j>1$, so $X$ must decompose.

(c) $\left[e_{i j, 0},\left[e_{i j, 0}, e_{i \ell, s}\right]\right]=0$ for $\ell=j \pm 1$ by Lemma 4

(d) $\left[e_{i 1,0},\left[e_{i 1,0}, e_{*, s}\right]\right]=0$. Computing $\left[e_{i 1,0}, e_{*, s}\right]$, one gets a contribution of $u_{X}$ for short exact sequences $0 \rightarrow \mathcal{O}(s \vec{c}) \rightarrow X \rightarrow S_{i 1} \rightarrow 0$, and the only possibility is $X \cong \mathcal{O}\left(s \vec{c}+\vec{x}_{i}\right)$. Then, computing $\left[e_{i 1,0},\left[e_{i 1,0}, e_{*, s}\right]\right]$, one gets a con- 
tribution of $u_{Y}$ for short exact sequences $0 \rightarrow \mathcal{O}\left(s \vec{c}+\vec{x}_{i}\right) \rightarrow Y \rightarrow S_{i j} \rightarrow 0$, but there are no nonsplit extensions.

(e) $\left[e_{*, 0},\left[e_{*, 0}, e_{i 1, s}\right]\right]=0$. Computing $\left[e_{*, 0}, e_{i 1, s}\right]$, one gets a contribution of $u_{X}$ for short exact sequences $0 \rightarrow \mathcal{O} \rightarrow X \rightarrow S_{i 1}\left[s w_{i}+1\right] \rightarrow 0$, and then one gets a contribution to $\left[e_{*, 0},\left[e_{*, 0}, e_{i 1, s}\right]\right]$ of $u_{Y}$ for short exact sequences $0 \rightarrow$ $X \rightarrow Y \rightarrow S_{i 1}\left[s w_{i}+1\right] \rightarrow 0$. Now by the theory of perpendicular categories [5], all of these sheaves belong to a subcategory of coh $\mathbb{X}$ corresponding to coherent sheaves on a weighted projective line with only one marked point, $a_{i}$, and for this subcategory it is known by the work of Geigle and Lenzing [4] that all indecomposable torsion-free sheaves have rank 1. But $Y$ would have to have rank 2.

(ix) $\left(\operatorname{ad} f_{u 0}\right)^{1-a_{u v}}\left(f_{v s}\right)=0$ for $u \neq v$. Similar to (viii).

\section{Proof of Theorem 1}

Let $\Phi$ be an additive group, (-, -) : $\Phi \times \Phi \rightarrow \mathbb{Z}$ a symmetric bilinear form, and let $\alpha \in \Phi$ satisfy $(\alpha, \alpha)=2$. One of the standard arguments in Lie theory shows that if $L$ is a $\Phi$-graded complex Lie algebra, $e \in L_{\alpha}, f \in L_{-\alpha}$ and $h=[e, f]$ have the property that ad $e$ and ad $f$ are locally nilpotent and ad $h$ acts on any $L_{\psi}$ as multiplication by $(\alpha, \psi)$, then $\operatorname{dim} L_{\phi}=\operatorname{dim} L_{\phi-(\alpha, \phi) \alpha}$ for any $\phi \in \Phi$. Namely, the operator $\theta=$ $\exp (\operatorname{ad} e) \exp (-\operatorname{ad} f) \exp (\operatorname{ad} e)$ is defined, and $\theta(h)=-h$. If $x \in L_{\phi}$, we can write $\theta(x)=\sum_{r \in \mathbb{Z}} y_{r}$ with $y_{r} \in L_{\phi+r \alpha}$, and

$$
\sum_{r \in \mathbb{Z}}(\alpha, \phi) y_{r}=\theta([h, x])=[\theta(h), \theta(x)]=[-h, \theta(x)]=\sum_{r \in \mathbb{Z}}-(\alpha, \phi+r \alpha) y_{r} .
$$

Thus, for all $r$ either $y_{r}=0$ or $(\alpha, \phi)=-(\alpha, \phi+r \alpha)$, so $r=-(\alpha, \phi)$. Thus, if $x \neq 0,(\alpha, \phi)$ must be an integer, and $\theta(x) \in L_{\phi-(\alpha, \phi) \alpha}$. Thus $\theta\left(L_{\phi}\right) \subseteq L_{\phi-(\alpha, \phi) \alpha}$. Similarly $\theta^{-1}\left(L_{\phi-(\alpha, \phi) \alpha}\right) \subseteq L_{\phi}$. This argument uses in several places that the base field has characteristic zero, but clearly it gives the following.

Lemma 9. Given a function $v: \Phi \rightarrow \mathbb{N}$ and $\phi \in \Phi$, there is some $\ell_{0}>0$ with the following property. If $L$ is a $\Phi$-graded Lie algebra over a field of characteristic $\ell \geq \ell_{0}$, and $e \in L_{\alpha}, f \in L_{-\alpha}$ and $h=[e, f]$ have the property that

$$
(\operatorname{ad} e)^{v(\psi)}(x)=0, \quad(\operatorname{ad} f)^{\nu(\psi)}(x)=0, \quad(\operatorname{ad} h)(x)=(\alpha, \psi) x
$$

for all $\psi \in \Phi$ and $x \in L_{\psi}$, then $\operatorname{dim} L_{\phi}=\operatorname{dim} L_{\phi-(\alpha, \phi) \alpha}$.

We are going to apply this lemma to Lie algebras of the form $L=L_{\Lambda}\left(\mathcal{R}_{\mathbb{X}}\right)$. They are graded by $\Phi=\hat{\Gamma}$ with $L_{0}=\Lambda \otimes_{\mathbb{Z}} \hat{\Gamma}$, and $u_{X} \in L_{\psi}$ and $u_{T X} \in L_{-\psi}$ for $X$ an indecomposable sheaf of type $\psi$.

Observe that if $\psi \neq 0$, then $\operatorname{dim} L_{\psi}$ is the number of indecomposable sheaves of type $\psi$ if $\psi \in \hat{\Gamma}_{+}$, is the number of indecomposable sheaves of type $-\psi$ if $-\psi \in \hat{\Gamma}_{+}$, and otherwise zero. 
The next lemma will ensure that the nilpotence conditions can be arranged. Since we have an inequality on the characteristic of the base field for $L$ in Lemma9, it is essential in the next lemma to have a uniform $v$, independent of the finite field $K$.

Lemma 10. Given a weight sequence $\mathbf{w}$ and vertex $v$, there is a function $v: \hat{\Gamma} \rightarrow \mathbb{N}$ such that for any weighted projective line $\mathbb{X}$ of type $\mathbf{w}$ over a finite field $K$, the Lie algebra $L=L_{\Lambda}\left(\mathcal{R}_{\mathbb{X}}\right)$ satisfies

$$
\left(\operatorname{ad} e_{v 0}\right)^{v(\psi)}(x)=\left(\operatorname{ad} f_{v 0}\right)^{v(\psi)}(x)=0
$$

for all $\psi \in \hat{\Gamma}$ and $x \in L_{\psi}$.

Proof. If $X, Y \in$ ind coh $\mathbb{X}, \operatorname{Ext}^{1}(X, X)=0$, and $u_{Z}$ is involved in $\left(\operatorname{ad} u_{X}\right)\left(u_{Y}\right)$, then $Z$ is the middle term of a nonsplit exact sequence whose end terms are $X$ and $Y$, so $\operatorname{dim} \operatorname{Ext}^{1}(X, Z)+\operatorname{dim} \operatorname{Ext}^{1}(Z, X)$ is strictly less than $\operatorname{dim} \operatorname{Ext}^{1}(X, Y)+\operatorname{dim}^{1} \operatorname{Ext}^{1}(Y, X)$. Thus $\left(\operatorname{ad} u_{X}\right)^{n}\left(u_{Y}\right)=0$ for $n>\operatorname{dim} \operatorname{Ext}^{1}(X, Y)+\operatorname{dim} \operatorname{Ext}^{1}(Y, X)$. The result now follows from Lemma 1] which still holds for $K$ finite, either by inspecting the argument, or by using the fact that an indecomposable sheaf of type $\phi$ splits over the algebraic closure of $K$ into summands which all have type $\phi / d$ for a positive integer $d$ dividing $\phi$.

Lemma 11. Suppose given a weight sequence $\mathbf{w}$, vertex $v$, and $0 \neq \phi \in \hat{\Gamma}_{+}$. For any prime $p$ there is a power $p^{n}$ such that if $\mathbb{X}$ is a weighted projective line of type $\mathbf{w}$ over a finite field $K$ which contains the field with $p^{n}$ elements, then the number of indecomposable sheaves of type $\phi$ is the same as the number of type $\pm s_{v}(\phi)$.

Proof. As explained above, let $\Phi=\hat{\Gamma}$ and let $\alpha=\alpha_{v}$. Let $v$ be given by the previous lemma, and $\ell_{0}$ by Lemma9.

Given $p$, choose $n$ so that $p^{n}-1$ is divisible by a prime $\ell \geq \ell_{0}$, and let $\Lambda$ be a field of characteristic $\ell$. As $K$ is a finite field containing the field with $p^{n}$ elements, we have $|K|=1$ in $\Lambda$, so the Lie algebra $L=L_{\Lambda}\left(\mathcal{R}_{\mathbb{X}}\right)$ is defined.

By Theorem 2 the elements $e=e_{v 0} \in L_{\alpha}$ and $f=f_{v 0} \in L_{-\alpha}$ satisfy $[e, f]=h_{v 0}$, and then by the definition of $L$ we have $\left(\operatorname{ad} h_{v 0}\right)(x)=(\alpha, \psi) x$ for $x \in L_{\psi}$. Thus we are in the setup of Lemma 9, so $\operatorname{dim} L_{\phi}=\operatorname{dim} L_{S_{v}(\phi)}$. This gives the result.

Now we work over an algebraically closed field. Given a weighted projective line $\mathbb{X}$ of weight type $\mathbf{w}$, and given $\phi \in \hat{\Gamma}_{+}$, one can find an algebraic variety $V$ with the action of a connected algebraic group $G$, in such a way that the orbits of $G$ on $V$ correspond 1-1 to isomorphism classes of certain sheaves on $\mathbb{X}$ of type $\phi$, including all indecomposable ones. Moreover, although the indecomposable sheaves need only form a constructible subset $V^{\text {ind }}$ of $V$, the subsets $V_{d}$ of $V$ consisting of the points whose $G$-orbit has dimension $d$ are locally closed in $V$, and $V_{d}^{\text {ind }}=V^{\text {ind }} \cap V_{d}$ is closed in $V_{d}$, hence an algebraic variety. One can then define the number of parameters of indecomposable sheaves of type $\phi$ to be

$$
n(\phi)=\max _{d}\left\{\operatorname{dim} V_{d}^{\text {ind }}-d\right\}
$$

and the number of top-dimensional families $t(\phi)$ can be defined as the sum over $d$ of the number of irreducible components of $V_{d}^{\text {ind }}$ of dimension $n(\phi)+d$. 
For representations of quivers this setup is explained in [10]. To convert parabolic bundles to quivers with relations, we used "squids" in [3. Lemma 5.5]. The essential extra ingredient here is Lemma 1, which ensures that there is some integer $N$ (depending only on $\mathbf{w}$ and $\phi$ ) such that any indecomposable parabolic bundle of type $\phi$ satisfies the following condition: $(*)$ if $E$ is the underlying vector bundle then the dual of the twist of $E$ by $N$ is generated by global sections. Twisting by $N$, the parabolic bundles satisfying $(*)$ correspond to representations of the squid satisfying certain conditions $(* *)$ mentioned in [3. Lemma 5.5]. Let $R(\alpha)$ denote the space of representations of the corresponding quiver of the appropriate dimension vector $\alpha$. The representations satisfying the relations for the squid define a closed subset of $R(\alpha)$, and then the representations satisfying ( $* *)$ define an open subset of that, and this is our variety $V$. An alternative approach would be to use canonical algebras, as in [4, §4] and [13, §3], and this would allow one to handle also the torsion sheaves. Another approach would be to use "quot-schemes". We omit the details as we only actually need the results for bundles, as the behaviour of the indecomposable torsion sheaves is trivial.

Lemma 12. Suppose given a weight sequence $\mathbf{w}$, vertex $v$, and $0 \neq \phi \in \hat{\Gamma}_{+}$. If $\mathbb{X}$ is a weighted projective line of weight type $\mathbf{w}$ over an algebraically closed field, then the number of parameters of indecomposable sheaves, and the number of top-dimensional families for type $\phi$ is the same as for type $\pm s_{v}(\phi)$. In particular, the number of isomorphism classes of indecomposable sheaves of type $\phi$ (a finite number or $\infty$ ) is the same as the number of type $\pm s_{v}(\phi)$.

Proof. This is essentially the same as in Kac's Theorem [7, 8] (see [10, §5]). We may assume that the point at infinity is not a marked point. Letting

$$
T=\mathbb{Z}\left[x_{1}, \ldots, x_{k}, \prod_{i<j}\left(x_{i}-x_{j}\right)^{-1}\right],
$$

any ring homomorphism $\theta: T \rightarrow K$ defines a weighted projective line $\mathbb{X}$ of weight type w over $K$ with marked points $\theta\left(x_{i}\right)$. Moreover the varieties constructed above are the $K$-points of suitable schemes of finite type over $T$.

Now $\operatorname{Ker} \theta$ is a prime ideal in $T$, and by constructibility results for dimensions of schemes, one obtains a maximal ideal $\mathfrak{m}$ lying over $\operatorname{Ker} \theta \operatorname{such}$ that the weighted projective lines over $K$ and over an algebraic closure of the finite field $T / \mathfrak{m}$ have the same numbers of parameters and top-dimensional families for types $\phi$ and $\pm s_{v}(\phi)$.

This reduces one to the case when $K$ is the algebraic closure of a finite field. Now if $K_{0}$ is a finite subfield containing the marked points, it suffices to show that over any finite field $K^{\prime}$ containing $K_{0}$, the numbers of $K^{\prime}$-points of the schemes for types $\phi$ and $s_{v}(\phi)$ correspond. This amounts to showing that the numbers of isomorphism classes of absolutely indecomposable sheaves of types $\phi$ and $s_{v}(\phi)$ are equal for the corresponding weighted projective line over $K^{\prime}$. By an argument involving minimal fields of definition (always containing $K_{0}$ ), it suffices to show that the numbers of indecomposable sheaves of types $\phi / d$ and $s_{v}(\phi / d)$ are equal for all $K^{\prime}$ containing $K_{0}$ and all positive integers $d$ dividing $\phi$. This follows from the last lemma, provided one takes $K_{0}$ large enough. 
Now let $\phi=\alpha+r \delta \in \hat{\Gamma}_{+}$. If $\alpha=0$ there are infinitely many indecomposables $S_{a}[r]$ of type $\phi$. If $\alpha$ is a real root, by a sequence of reflections one reduces to $\pm \alpha_{v}+r \delta$, when there is a unique indecomposable. If $\alpha$ is an imaginary root, one reduces to $\alpha+r \delta$ with $\alpha$ in the fundamental region, and there are infinitely many indecomposables by [3, Lemma 5.6]. If $\alpha$ is not a root, one reduces to the case when $\alpha$ is not positive or negative, or has disconnected support, and there is no indecomposable. This completes the proof of Theorem 1. Finally, note that the same method gives a new proof of Kac's Theorem in the original case of quivers.

\section{References}

[1] Atiyah, M. F.: Vector bundles over an elliptic curve. Proc. London Math. Soc. 7, 414-452 (1957) Zbl 0084.17305 MR 0131423

[2] Baumann, P., Kassel, C.: The Hall algebra of the category of coherent sheaves on the projective line. J. Reine Angew. Math. 533, 207-233 (2001) Zbl 0967.18005 MR 1823869

[3] Crawley-Boevey, W.: Indecomposable parabolic bundles and the existence of matrices in prescribed conjugacy class closures with product equal to the identity. Publ. Math. Inst. Hautes Études Sci. 100, 171-207 (2004) Zbl 1065.14040 MR 2102700

[4] Geigle, W., Lenzing, H.: A class of weighted projective curves arising in representation theory of finite dimensional algebras. In: Singularities, Representations of Algebras, and Vector Bundles (Lambrecht, 1985), G.-M. Greuel and G. Trautmann (eds.), Lecture Notes in Math. 1273, Springer, Berlin, 265-297 (1987) Zbl 0651.14006 MR 0915180

[5] Geigle, W., Lenzing, H.: Perpendicular categories with applications to representations and sheaves. J. Algebra 144, 273-343 (1991) Zbl 0748.18007 MR 1140607

[6] Hubery, A.: From triangulated categories to Lie algebras: a theorem of Peng and Xiao. In: Trends in Representation Theory of Algebras and Related Topics (Querétaro, 2004), J. A. de la Peña and R. Bautista (eds.), Contemp. Math. 406, Amer. Math. Soc., Providence, RI, 51-66 (2006) Zbl 1107.16021 MR 2258041

[7] Kac, V. G.: Infinite root systems, representations of graphs and invariant theory. Invent. Math. 56, 57-92 (1980) Zbl 0427.17001 MR 0557581

[8] Kac, V. G.: Root systems, representations of quivers and invariant theory. In: Invariant Theory (Montecatini, 1982), F. Gherardelli (ed.), Lecture Notes in Math. 996, Springer, Berlin, 74108 (1983) Zbl 0534.14004 MR 0718127

[9] Kapranov, M. M.: Eisenstein series and quantum affine algebras. J. Math. Sci. (New York) 84, 1311-1360 (1997) Zbl 0929.11015 MR 1465518

[10] Kraft, H., Riedtmann, C.: Geometry of representations of quivers. In: Representations of Algebras (Durham, 1985), P. Webb (ed.), London Math. Soc. Lecture Note Ser. 116, Cambridge Univ. Press, 109-145 (1986) Zbl 0632.16019 MR 0897322

[11] Lenzing, H.: Representations of finite dimensional algebras and singularity theory. In: Trends in Ring Theory (Miskolc, 1996), Canad. Math. Soc. Conf. Proc. 22, Amer. Math. Soc., Providence, RI, 71-97 (1998) Zbl 0895.16003 MR 1491919

[12] Lenzing, H., Meltzer, H.: Sheaves on a weighted projective line of genus one, and representations of a tubular algebra. In: Representations of Algebras (Ottawa, 1992), Canad. Math. Soc. Conf. Proc. 14, Amer. Math. Soc., Providence, RI, 313-337 (1993) Zbl 0809.16012 MR 1265294

[13] Lenzing, H., de la Peña, J. A.: Wild canonical algebras. Math. Z. 224, 403-425 (1997) Zbl 0882.16011 MR 1439198 
[14] Lin, Y., Peng, L.: Elliptic Lie algebras and tubular algebras. Adv. Math. 196, 487-530 (2005) Zbl 1127.17025 MR 2166313

[15] Moody, R. V., Eswara Rao, S., Yokonuma, T.: Toroidal Lie algebras and vertex representations. Geom. Dedicata 35, 283-307 (1990) Zbl 0704.17011 MR 1066569

[16] Peng, L., Xiao, J.: Root categories and simple Lie algebras. J. Algebra 198, 19-56 (1997) Zbl 0893.16007 MR 1482975

[17] Peng, L., Xiao, J.: Triangulated categories and Kac-Moody algebras. Invent. Math. 140, 563603 (2000) Zbl 0966.16006 MR 1760751

[18] Ringel, C. M.: Tame Algebras and Integral Quadratic Forms. Lecture Notes in Math. 1099, Springer, Berlin (1984) Zbl 0546.16013 MR 0774589

[19] Schiffmann, O.: Noncommutative projective curves and quantum loop algebras. Duke Math. J. 121, 113-168 (2004) Zbl 1054.17021 MR 2031167 